Supporting Information

\title{
Broad-Band Photodetectors Based on Copper Indium Diselenide Quantum Dots in a Methylammonium Lead Iodide Perovskite Matrix
}

Zonghui Duan, ${ }^{\dagger}$ Jiajia Ning, ${ }^{\dagger}$ Mengyu Chen, ${ }^{\ddagger}$ Yuan Xiong, ${ }^{\dagger}$ Wenhong Yang, ${ }^{*}$ Fengping Xiao, ${ }^{\dagger}$ Stephen V. Kershaw, ${ }^{\dagger}$ Ni Zhao, ${ }^{\ddagger}$ Shumin Xiao, ${ }^{\#}$ Andrey L. Rogach ${ }^{1,}$

*E-mail: andrey.rogach@cityu.edu.hk

${ }^{\dagger}$ Department of Materials Science and Engineering, and Centre for Functional Photonics (CFP), City University of Hong Kong, 83 Tat Chee Avenue, Kowloon, Hong Kong SAR 999077, China

${ }^{\ddagger}$ Department of Electronic Engineering, Chinese University of Hong Kong, New Territories, Hong Kong SAR 999077, China

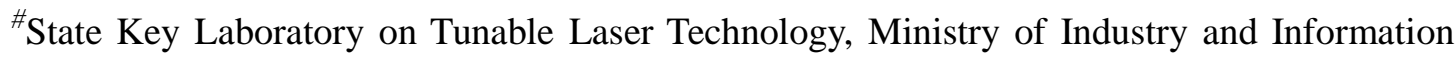
Technology Key Lab of Micro-Nano Optoelectronic Information System, Shenzhen Graduate School, Harbin Institute of Technology, 518055 Shenzhen, China 


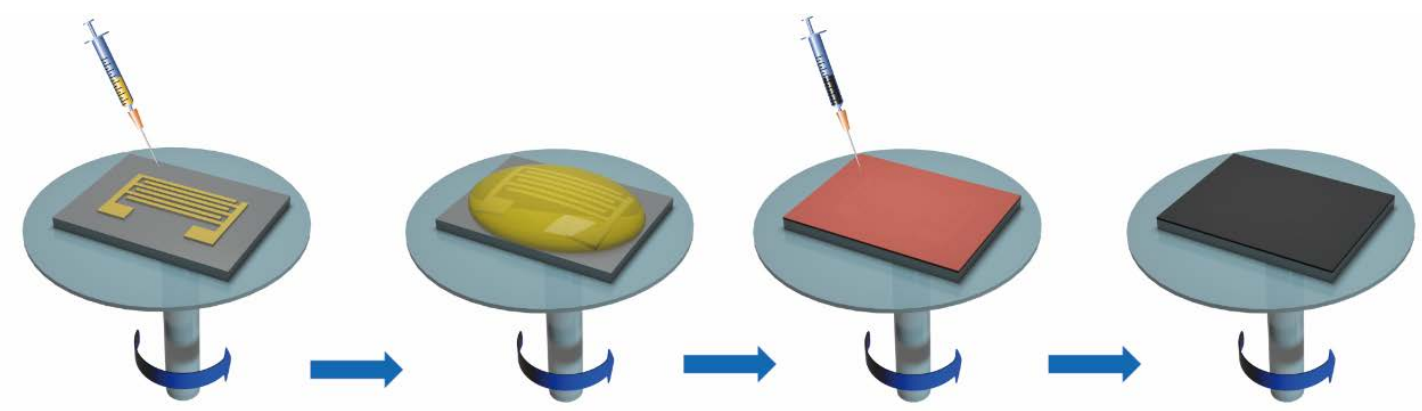

Figure S1. Schematic illustration of film and device preparation procedures.

(a)

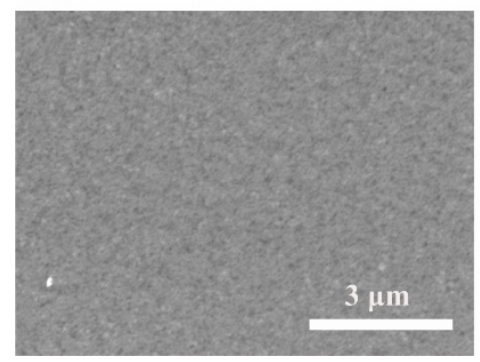

(b)

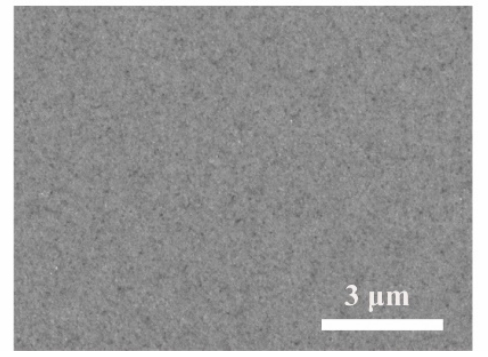

(c)

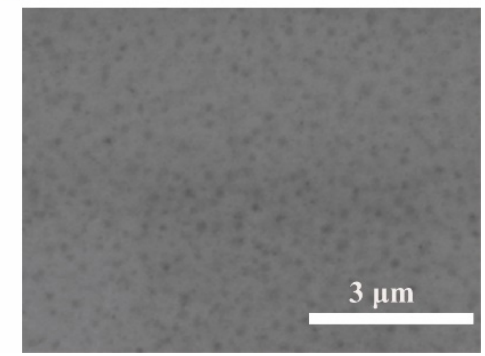

Figure S2. SEM images of the $\mathrm{MAPbI}_{3}$ films treated with a) $2.4 \% \mathrm{w} / \mathrm{v}, \mathrm{b}$ ) $4 \% \mathrm{w} / \mathrm{v}$, and c) $8 \% \mathrm{w} / \mathrm{v}$ of CISe QDs, respectively.
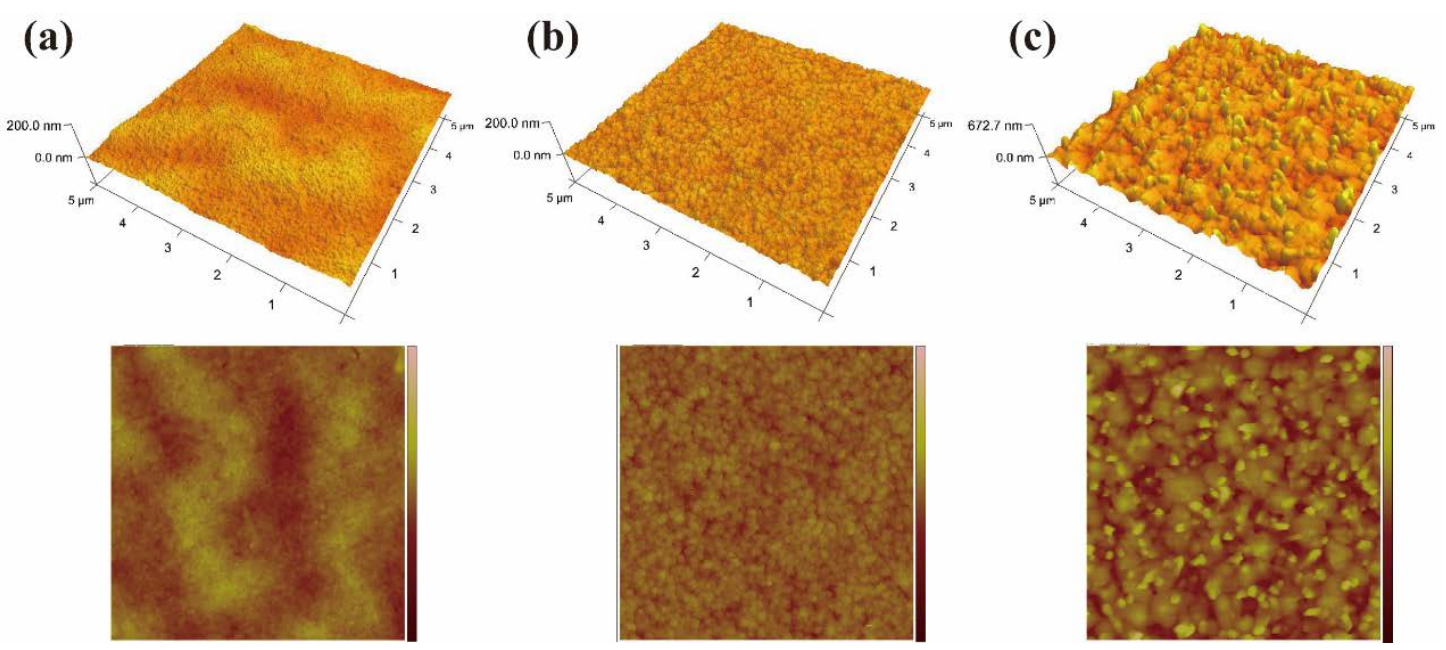

Figure S3. AFM images of a) $\mathrm{MAPbI}_{3} / \mathrm{CISe} 2.4 \%$, b) $\mathrm{MAPbI}_{3} / \mathrm{CISe} 4 \%$ and c) $\mathrm{MAPbI}_{3} / \mathrm{CISe} \%$ hybrid films. 


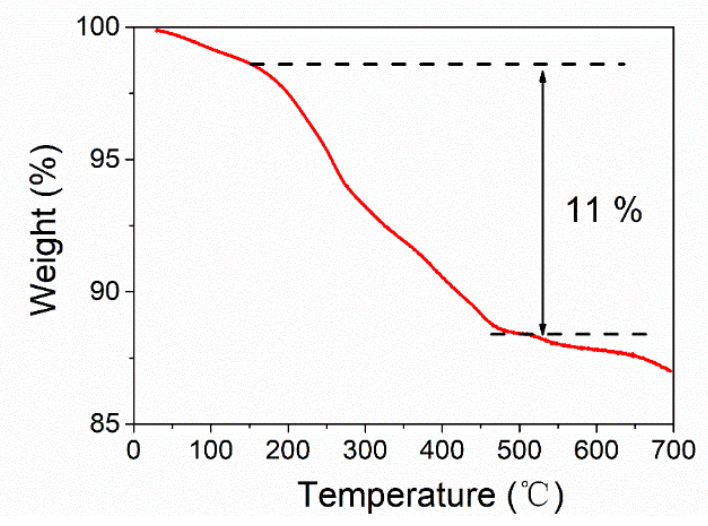

Figure S4. TGA curve of CISe QDs in air.

(a)

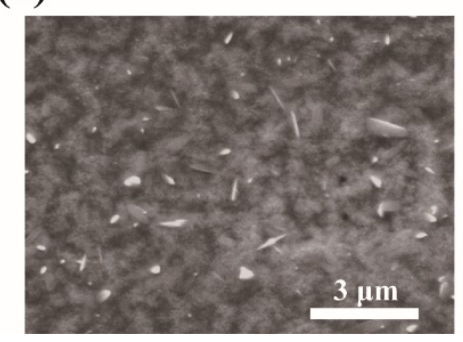

(b)

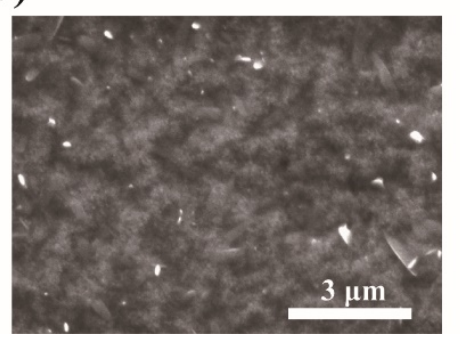

(c)

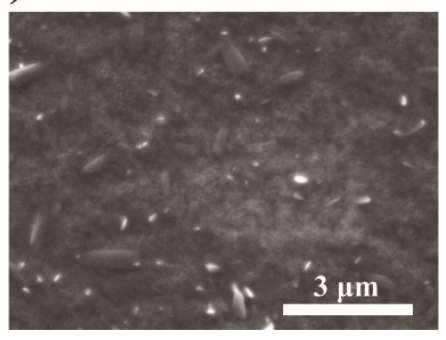

Figure S5. a) SEM images of the $\mathrm{MAPbI}_{3}$ films treated with the same amounts of OLA ligands as used in the a) $2.4 \% \mathrm{w} / \mathrm{v}$, b) $4 \% \mathrm{w} / \mathrm{v}$, and c) $5.6 \% \mathrm{w} / \mathrm{v}$ CISe QD solutions, respectively, but with the QDs absent.

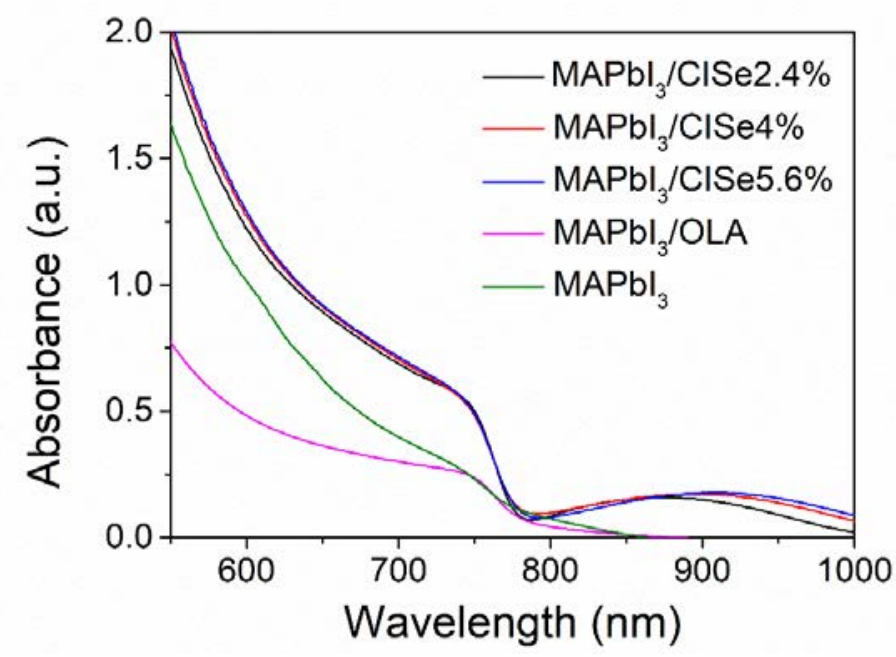

Figure S6. Absorption spectra of the bare $\mathrm{MAPbI}_{3}$ film, and the $\mathrm{MAPbI}_{3}$ films treated with different concentrations of CISe QDs or OLA, as indicated. 
(a)

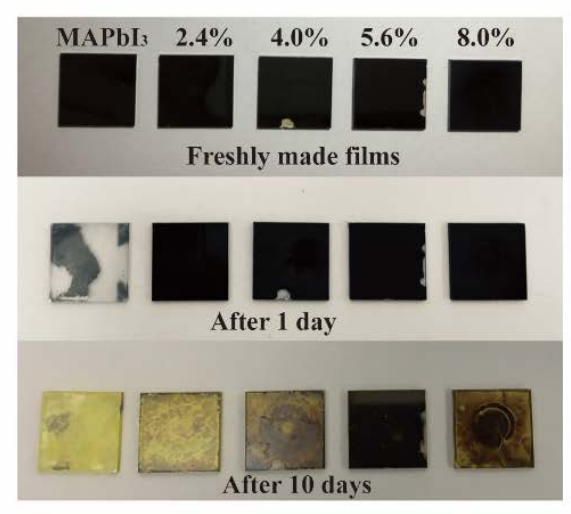

(b)

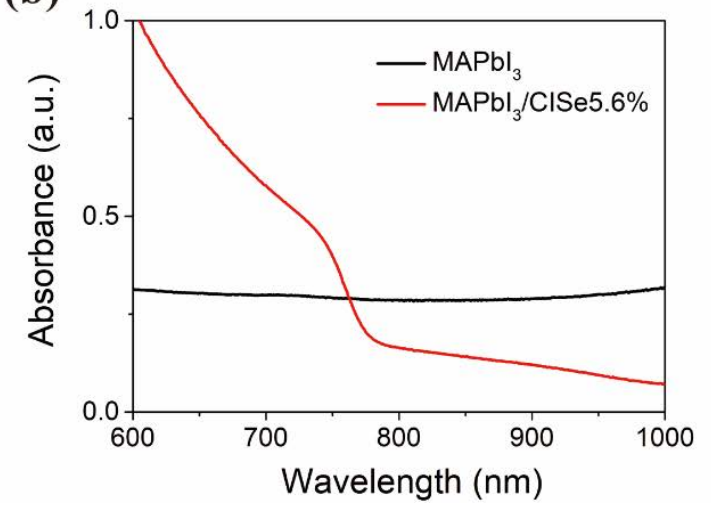

Figure S7. a) Photographs of the $\mathrm{MAPbI}_{3}$ and $\mathrm{MAPbI}_{3} / \mathrm{CISe}$ hybrid films with different concentrations of CISe QDs, freshly made and after storage for 1 and 10 days. b) Absorption spectra of the $\mathrm{MAPbI}_{3}$ and $\mathrm{MAPbI}_{3} / \mathrm{CISe} 4 \%$ films which were exposed to the ambient environment for 10 days.

(a)

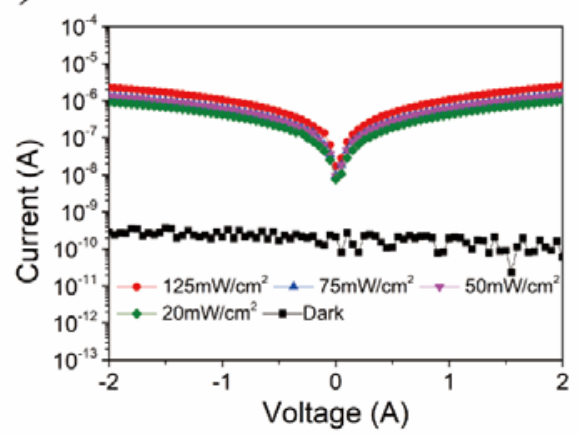

(b)

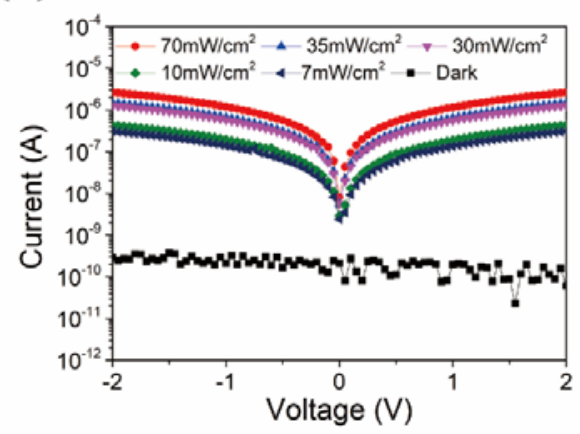

Figure S8. Logarithmic form of the current-voltage (I-V) curves of the $\mathrm{MAPbI}_{3} / \mathrm{CISe} 5.6 \%$-based photodetectors operating between $-2 \mathrm{~V}$ to $2 \mathrm{~V}$, collected under a) $405 \mathrm{~nm}$ and b) $675 \mathrm{~nm}$ light illumination with a range of power intensities as indicated on the frames. 

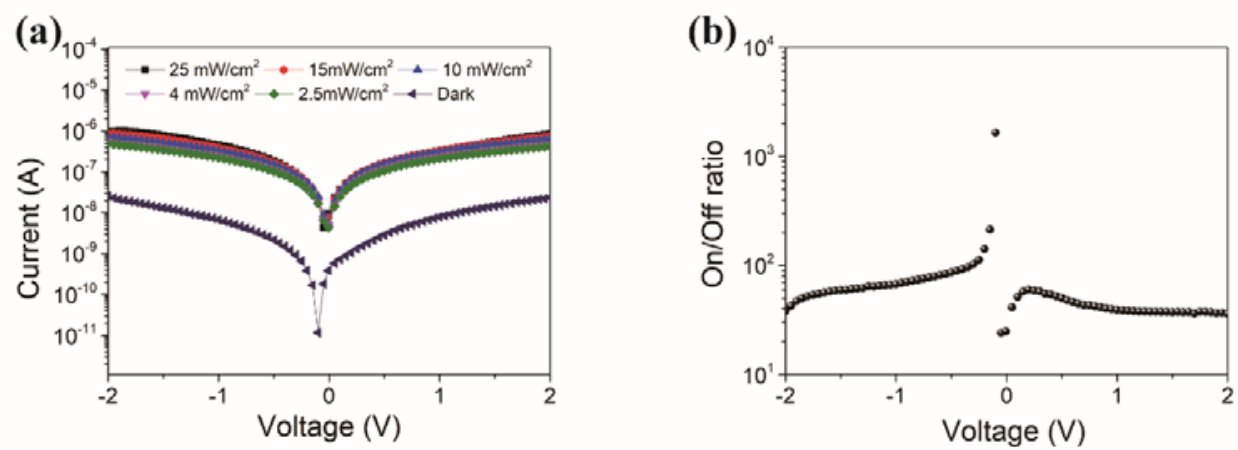

Figure S9. a) Logarithmic form of the current-voltage (I-V) curves of the $\mathrm{MAPbI}_{3}$ device treated with 5.6\% w/v CISe QDs solution where the standard OLA pre-treatment was applied. b) On-off ratio of the photodetector based on the $\mathrm{MAPbI}_{3}$ film treated with OLA.

(a)

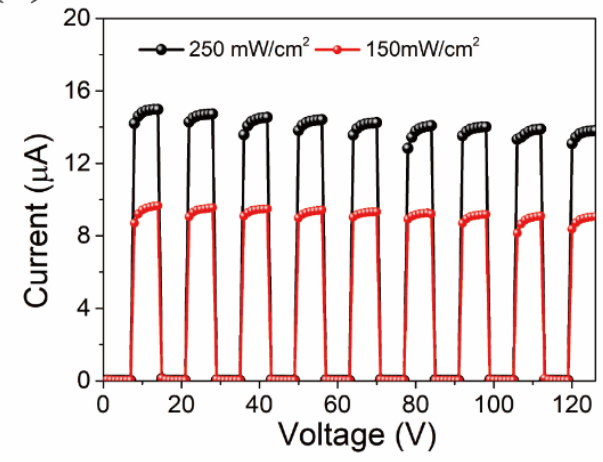

(b)

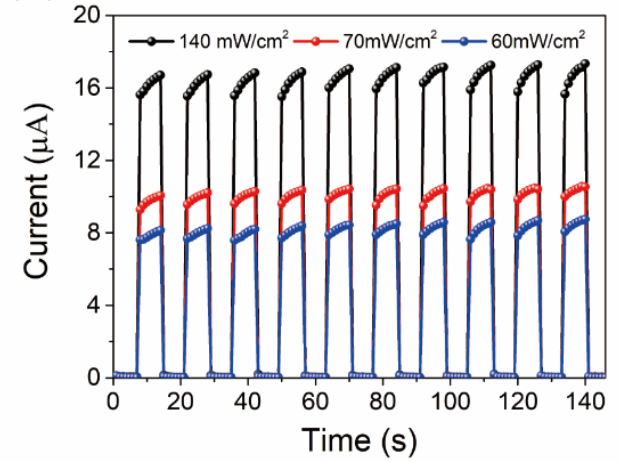

Figure S10. Time-dependent current of the $\mathrm{MAPbI}_{3} / \mathrm{CISe} 5.6 \%$ devices at a) $405 \mathrm{~nm}$ and b) $675 \mathrm{~nm}$ light illumination under $2 \mathrm{~V}$ bias in the ambient environment. The respective power densities for each measurement are indicated on the frames. 
(a)

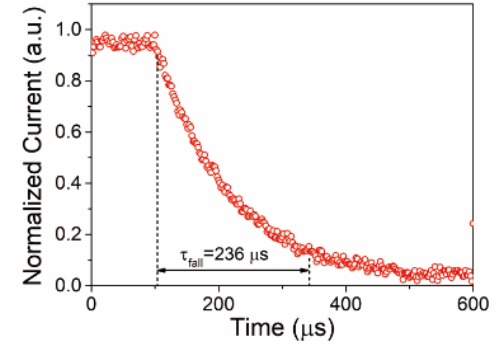

(b)

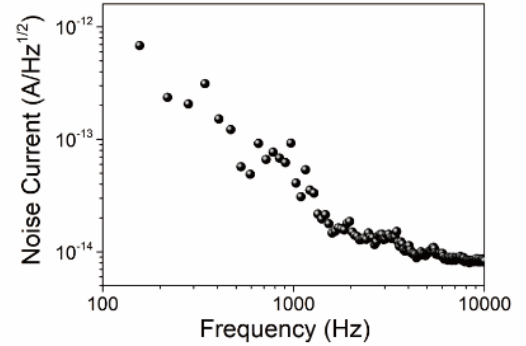

(c)

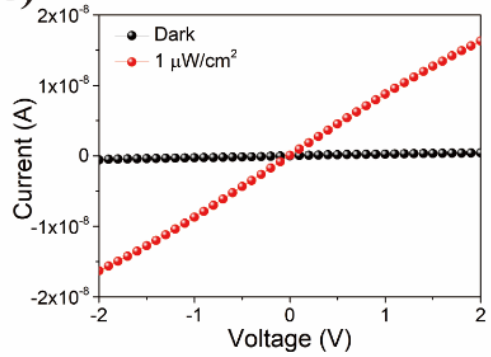

Figure S11. a) Transient current of the $\mathrm{MAPbI}_{3} / \mathrm{CISe} 5.6 \%$ photodetector. b) Noise current of the $\mathrm{MAPbI}_{3} / \mathrm{CISe} 5.6 \%$ photodetector at $1.5 \mathrm{~V}$. c) $\mathrm{I}-\mathrm{V}$ curves of the $\mathrm{MAPbI}_{3} / \mathrm{CISe} 5.6 \%$ device measured under dark and under $525 \mathrm{~nm}$ light illumination with a power density of $1 \mu \mathrm{W} / \mathrm{cm}^{2}$.

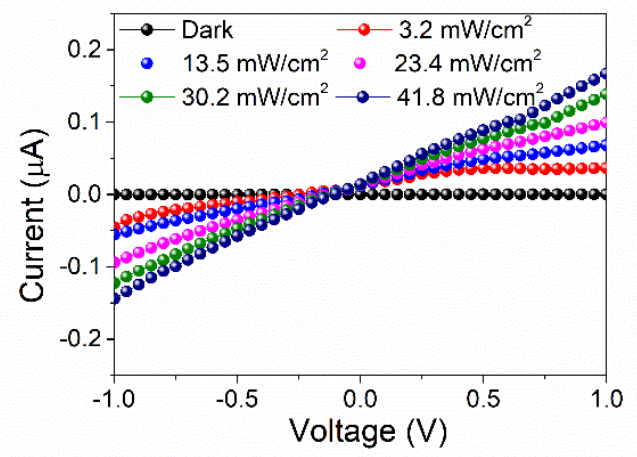

Figure S12. I-V curves of the $\mathrm{MAPbI}_{3} / \mathrm{CISe} 5.6 \%$ device measured under $880 \mathrm{~nm}$ laser illumination.

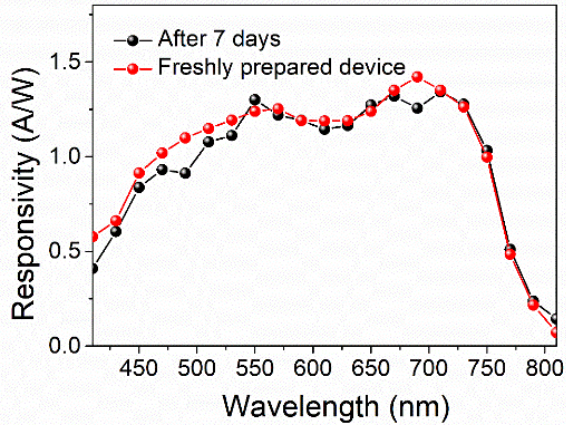

Figure S13. Responsivity of the $\mathrm{MAPbI}_{3} / \mathrm{CISe} 5.6 \%$-based photodetector measured just after fabrication and after exposure to the ambient environment for 7 days. 